\title{
Variation in nebulizer output and weight output from Mefar dosimeter: implications for multicentre studies
}

\section{To the Editor:}

We wish to comment upon the paper of CHINN et al. [1] in which both weight and aerosol output data were given for 193 Mefar nebulizers from 27 centres involved in the European Community Respiratory Health Survey (ECRHS). A single centre in Turin determined nebulizer output using the sodium fluoride $(\mathrm{NaF})$ tracer method described by DenNis et al. [2]. The weight output was given as $11 \mathrm{mg} \cdot \mathrm{s}^{-1}$ with a coefficient of variation of $5.8 \%$. Aerosol output (AO), as a percentage of weight output gave a mean of $43 \%$ (i.e. mean $\mathrm{AO}$ of approximately $4.7 \mathrm{mg} \cdot \mathrm{s}^{-1}$ ) with a between-centre range of $40-52 \%$ (AO range $4.4-5.7 \mathrm{mg} \cdot \mathrm{s}^{-1}$ ).

We regard these data from the single Turin centre with some concern, in view of our experience with similar Mefar nebulizers, which is supported by published data by DenNis et al. [3], suggesting a considerably higher AO. They found a similar weight output to that given by CHINN et al. [1] but a considerable betweenbatch variation in Mefar nebulizer output, with mean aerosol output between batches ranging from 5.7-10.6 $\mathrm{mg} \cdot \mathrm{s}^{-1}$ (AO 51-75\%).

We recently re-analysed the outputs of a batch of Mefar nebulizers used in the ECRHS. We found a mean $\mathrm{AO}$ of $6.7 \mathrm{mg} \cdot \mathrm{s}^{-1}$ (AO 61.4\%). A batch of unused Mefar nebulizers analysed at the same time, had an AO of 7.1 $\mathrm{mg} \cdot \mathrm{s}^{-1}$ (AO 65.3\%). Furthermore, we recently supervised the calibration of a batch of 10 Mefar nebulizers by the NaF method by a third centre (Freeman Hospital, Newcastle Upon Tyne; unpublished data) and obtained a mean $\mathrm{AO}$ of $6.9 \mathrm{mg} \cdot \mathrm{s}^{-1}$. It seems unlikely that the aerosol outputs from five batches of Mefar nebulizers, as measured by DENNIs et al. [3] and ourselves, would differ so much from the outputs reported by CHINN et al. [1]. It would also seem unlikely that a modern welldesigned nebulizer system would have such a poor AO (43\%) as that described by CHINN et al. [1]. One has to wonder whether this represents some systematic methodological artefact and it is also our experience that $\mathrm{NaF}$ nebulizer calibration is technically difficult even with extensive experience.

Could we suggest that a number of batches of nebulizers used in the ECRHS survey be sent to ourselves and others with substantial experience of measuring aerosol output, so that the data presented by CHINN et al. [1] can be corroborated? It is vital to the credibility of the airway reactivity measurements made in the ECRHS survey that true aerosol output be known, and that potential between-batch variability is fully established and quantified.

\section{References}

1. Chinn S, Arosa WA, Jarvis DL, Luczynska CM, Burney PGJ. Variation in nebuliser output and weight output from Mefar dosimeter: implications for multicentre studies. Eur Respir J 1997; 10: 452-456.

2. Dennis JH, Stenton SC, Beach JR, Avery AJ, Walters EH, Hendrick DJ. Jet and ultrasonic nebuliser output: use of a new method for direct measurements of aerosol output. Thorax 1990; 45: 728-732.

3. Dennis JH, Avery AJ, Walters EH, Hendrick DJ. Calibration of aerosol output from Mefar dosimeter implications for epidemiological studies. Eur Respir J 1992; 5: 1279-1282.

\section{R.J. Ward, D.W. Reid, E.H. Walters}

The Alfred Healthcare Group, Dept of Respiratory Medicine, Melbourne, Victoria 3181, Australia.

\section{REPLY}

From the authors:

We welcome the opportunity to reply to the letter from Ward et al. We are surprised at the implied criticism of our paper [1], that nebulizer aerosol output was measured in a single laboratory. The aim was to examine the within- and between-centre variation in the European Community Respiratory Health Survey (ECRHS), not between-laboratory variation in calibration, and clearly our aim required measurements made under identical conditions. As Melbourne was not one of the centres in the ECRHS that returned nebulizers for central testing we are unable to comment on whether the Melbourne batch was also an outlier, as were the nebulizers received from Bergen, Norway, or whether there was a systematic difference between results from the Turin and Melbourne laboratories. The latter would not invalidate the conclusion drawn in our paper that the within-centre variation in nebulizer output had a coefficient of variation of less than $10 \%$.

Even if it were possible to adjust the methacholine challenge data for aerosol output of the nebulizers used, this would not greatly enhance the information provided. 
"True" aerosol output is not the only factor affecting the dose of methacholine delivered to the lung or bronchi, and concentration on this one aspect of the challenge protocol is unhelpful. Our paper was prompted by concerns about between-batch variation in nebulizer output and presented a new summary statistic, log-slope, that is independent of between-batch variation. The ECRHS data have been analysed using both provocative dose causing a $20 \%$ fall in forced expiratory volume in one second and log-slope to show that the results are not sensitive to the concerns raised.

\section{Reference}

1. Chinn S, Arossa WA, Jarvis DL, Luczynska CM, Burney PGJ. Variation in nebulizer aerosol output and weight output from the Mefar dosimeter: implications for multicentre studies. Eur Respir J 1997: 10; 452-456.

S. Chinn, W.A. Arosa, D.L. Jarvis, C.M. Luczynska, P.G.J. Burney

Dept of Public Health Medicine, United Medical and Dental Schools, St Thomas' Campus, London SE1 1EH, UK. 